\title{
A re-characterization of the Kemeny distance
}

\author{
Citation for published version (APA):
}

Can, B., \& Storcken, A. J. A. (2013). A re-characterization of the Kemeny distance. Maastricht University, Graduate School of Business and Economics. GSBE Research Memoranda No. 009 https://doi.org/10.26481/umagsb.2013009

Document status and date:

Published: 01/01/2013

DOI:

10.26481/umagsb.2013009

Document Version:

Publisher's PDF, also known as Version of record

\section{Please check the document version of this publication:}

- A submitted manuscript is the version of the article upon submission and before peer-review. There can be important differences between the submitted version and the official published version of record.

People interested in the research are advised to contact the author for the final version of the publication, or visit the DOI to the publisher's website.

- The final author version and the galley proof are versions of the publication after peer review.

- The final published version features the final layout of the paper including the volume, issue and page numbers.

Link to publication

\footnotetext{
General rights rights.

- You may freely distribute the URL identifying the publication in the public portal. please follow below link for the End User Agreement:

www.umlib.nl/taverne-license

Take down policy

If you believe that this document breaches copyright please contact us at:

repository@maastrichtuniversity.nl

providing details and we will investigate your claim.
}

Copyright and moral rights for the publications made accessible in the public portal are retained by the authors and/or other copyright owners and it is a condition of accessing publications that users recognise and abide by the legal requirements associated with these

- Users may download and print one copy of any publication from the public portal for the purpose of private study or research.

- You may not further distribute the material or use it for any profit-making activity or commercial gain

If the publication is distributed under the terms of Article $25 \mathrm{fa}$ of the Dutch Copyright Act, indicated by the "Taverne" license above, 


\section{Maastricht University}

Burak Can, Ton Storcken

A re-characterization of the Kemeny distance

RM/ 13/009

\section{GSBE}

Maastricht University School of Business and Economics

Graduate School of Business and Economics

P.O Box 616

NL- 6200 MD Maastricht

The Netherlands 


\title{
A re-characterization of the Kemeny distance
}

\author{
Burak Can* Ton Storcken ${ }^{\dagger}$
}

February 2013

\begin{abstract}
The well-known swap distance (Kemeny (1959); Kendall (1938); Hamming (1950) $)$ is analyzed. On weak preferences, this function was characterized by Kemeny (1959) with five conditions; metric, betweenness, neutrality, reducibility, and normalization. We show that the same result can be achieved without the reducibility condition, therefore, the original five conditions are not logically independent. We provide a new and logically independent characterization of the Kemeny distance and provide some insight to further analyze distance functions on preferences.
\end{abstract}

JEL Classification: D63, D71, D72

Keywords: Kemeny distance, swap distance, inversion metric, preferences

\section{Introduction}

The use of a distance function in comparing preferences, rankings, orders, lists, queue's etc. to indicate similarities or differences between these objects is a common practice. A natural and frequently used way in measuring distances between any two such objects is by counting the number of minimal swaps necessary to invert one into the other. Within this context, the reader may be familiar with the following (most probably non-exhaustive) list of names: the Kemeny (1959) distance, the Kendall-tau (1938) distance, swap distance, bubble-sort distance, inversion metric, word metric, permutation swap, the Damerau Levenshtein $(1964,1966)$ distance, the Hamming (1950) distance, and so forth. In fact, prior to Kemeny (1959) and Kendall (1938), the use of this distance can even be traced back to Cramer (1750).

Kemeny (1959) characterized this function on the domain of weak orders. From here on we shall call this metric function, the Kemeny distance. The characterization involved five conditions: metric, betweenness, neutrality, normalization and reducibility. The "metric" condition ensures that the function to be characterized is indeed a distance measure in mathematical terms. "Betweenness" requires that an order which is considered between two other orders is also on a shortest path

\footnotetext{
${ }^{*}$ Corresponding author: Department of Economics, School of Business and Economics, Maastricht University, P.O. Box 616, 6200 MD Maastricht, the Netherlands; E-mail: b.can@maastrichtuniversity.nl, Phone number: +31 43 3883807. Authors are grateful to Bettina Klaus, Kristof Bosmans, M. Remzi Sanver and Uğur Özdemir for their comments on earlier drafts and three anonymous reviewers of CoopMAS 2013 Workshop. The support of Netherlands Organisation for Scientific Research (NWO, Open Competitie) under the grant with project nr. 400-09354 , is gratefully acknowledged.

${ }^{\dagger}$ Department of Quantitative Economics, School of Business and Economics, Maastricht University, P.O. Box 616, NL 6200 MD Maastricht, the Netherlands; E-mail: t.storcken@maastrichtuniversity.nl, Phone number: +31 43 3883728, Fax number:+31 433884874
} 
between these two. "Neutrality" states that the names of the ordered alternatives have no influence on the distances between these orders. "Normalization" sets the minimal measurable distance to real number 1. Finally, "reducibility" imposes that the distance only depends on those parts where orders differ.

The characterization of the Kemeny distance is published in Kemeny (1959), yet the proof can be found in Kemeny and Snell (1962). To the best of the authors' knowledge, a firm discussion on the independence of these five conditions, however, cannot be found in literature. Both in Kemeny and Snell (1962) and in later works on distances between orders (see Bogart (1973) for a thorough analysis), this subject is not investigated. In this paper, we show that the Kemeny distance is the only function that satisfies metric, betweenness, neutrality and normalization. Hence, we provide a new characterization of a well-known measure with fewer conditions. As a consequence, these four conditions imply reducibility, which is then not needed in the characterization.

This paper proceeds as follows. In Section 2, we present the model and the notation. Section 3 introduces distance functions and conditions on these functions. In Section 4 we provide the new characterization of the Kemeny distance. In Section 5 we discuss the logical independence of the conditions and provide a brief discussion of the results.

\section{Model}

Let $\mathcal{A}$ denote a finite set of alternatives, i.e. the universal set of alternatives. Let $P(\mathcal{A})$ denote the set of all nonempty subsets of $\mathcal{A}$. Given a set $A \in P(\mathcal{A})$, preferences are modeled by weak orders, i.e., complete, transitive binary relations over $A$ whereas strict preferences are modeled by linear orders, i.e., complete, transitive and antisymmetric binary relations over $A$. The set of all preferences over $A$ is denoted by $\mathcal{W}^{A}$ whereas the set of strict preferences is denoted by $\mathcal{L}^{A}$. Note that $\mathcal{L}^{A} \subseteq \mathcal{W}^{A}$ for all $A \in P(\mathcal{A})$. As a generic preference (possibly strict), we use $R \in \mathcal{W}^{A}$. In the sequel, we use $\mathcal{W}$ (or $\mathcal{L}$ ) rather than $\mathcal{W}^{A}$ (or $\mathcal{L}^{A}$ ) whenever it is clear over which subsets these weak (or linear) orders are defined.

Let $R$ be a weak order on $A$ and let $x$ and $y$ be alternatives in $A$. Then $(x, y) \in R$ has the usual interpretation: " $x$ is ordered at least as good as $y$ at $R$ ". In case $(y, x)$ is also in $R$ we say that both are ordered equally good. Where in case $(y, x)$ is not in $R$ we say that " $x$ is ordered above $y$ " or " $y$ is ordered below $x$ ". Given a linear order $R \in \mathcal{L}$ and two distinct alternatives $x, y \in A$, we write $R=\ldots x \ldots y \ldots$ if $(x, y) \in R$, and $R=\ldots x y \ldots$ if additionally there exists no $z \in A \backslash\{x, y\}$ such that $(x, z) \in R$ and $(z, y) \in R$, i.e., $x$ and $y$ are "consecutively" ordered in $L$.

For any $A, B \in P(\mathcal{A})$ with $B \subseteq A$ and for any $R \in \mathcal{W}^{A}$, we write $R_{B} \in \mathcal{W}^{B}$ to denote the order restricted to $B$, i.e., $R_{B}=R \cap(B \times B)$. The strict upper contour set of $x$ in $R$ is defined by $U C(x, R)=\{z \in A \mid(z, x) \in R$ and $(x, z) \notin R\}$, i.e., the set of alternatives that are ordered above $x$ in $R$. Similarly $L C(x, R)=\{z \in A \mid(x, z) \in R$ and $(z, x) \notin R\}$ denotes the strict lower contour set of $x$ in $R$. Then $R_{U C(x)}$ denotes order $R$ restricted to the upper contour set of $x$ in $R$ and $R_{L C(y)}$ denotes the restriction of $R$ to the lower contour set of $y$ in $R$. Taking $[x, y]=A \backslash(U C(x, R) \cup L C(y, R))$ then $R_{[x, y]}$ denotes the restriction of $R$ to all alternatives which are ordered weakly between $x$ and $y$, e.g., for $A=\{a, b, c, d, e, f\}$ and $R=a b c d e f$; we have $R_{U C(c)}=a b, R_{L C(c)}=\operatorname{def}$ and $R_{[c, e]}=c d e$.

Given $A \in P(\mathcal{A})$, and $l=1,2, \ldots,|A|$, the alternative in the $l^{t h}$ position in a linear order $R \in \mathcal{L}$ 
is denoted by $R(l)$, e.g. $R(1)$ is the top alternative in $R$, and $R(2)$ is the one ranked below $R(1)$. Let $k$ be a number smaller than $|A|$. Then linear orders $R, R^{\prime} \in \mathcal{L}$ form an elementary change in position $k$ whenever $R(k)=R^{\prime}(k+1), R^{\prime}(k)=R(k+1)$ and for all $t \in\{1,2, \ldots,|A|\} \backslash\{k, k+1\}$ we have $R(t)=R^{\prime}(t)$. So, $R$ and $R^{\prime}$ can be inverted into one another by one single swap of $(R(k), R(k+1))$, i.e., a swap of alternatives on the $k^{t h}$ and the $(k+1)^{t h}$ positions.

Given any two preferences $R, R^{\prime} \in \mathcal{W}$, we say $R^{\prime \prime}$ is between $R$ and $R^{\prime}$ whenever $R \cap R^{\prime} \subseteq$ $R^{\prime \prime} \subseteq R \cup R^{\prime}$. Note that if preferences are strict, e.g., $P, P^{\prime}, P^{\prime \prime} \in \mathcal{L}$, the definition is reduced to $P \cap P^{\prime} \subseteq P^{\prime \prime}$ where $P^{\prime \prime}$ is between $P$ and $P^{\prime}$. Given any sequence of preferences $R^{1}, \ldots, R^{t} \in \mathcal{W}$, we say the preferences $R^{1}, \ldots, R^{t}$ are "on a line" and denote as $\left[R^{1}, \ldots, R^{t}\right]$ if $R^{j}$ is between $R^{i}$ and $R^{k}$ for all $1 \leq i \leq j \leq k \leq t$.

\section{Distance functions and conditions}

A metric - or a distance - function assigns a nonnegative value to pairs of preferences, which is interpreted as the dissimilarity between two preferences. We consider metrics that compare preferences over identical subset: ${ }^{1}$ of alternatives, e.g., $\delta: \mathcal{W}^{A} \times \mathcal{W}^{A} \rightarrow \mathbb{R}_{+}$. From this point on we abuse notation and instead of repeating for all $A \in P(\mathcal{A})$ and for all $R \in \mathcal{W}^{A}$, we simply write for all $R \in \mathcal{W}$. This is in line with the convention in the proof of Kemeny and Snell (1962). Similarly, a function $\delta: \mathcal{W} \times \mathcal{W} \rightarrow \mathbb{R}$, is called a metric (or a distance) function if and only if it satisfies the following metric conditions (Condition 1):

Condition 1: (Metric conditions): For all $R, R^{\prime}, R^{\prime \prime} \in \mathcal{W}$,

i) Non-negativity: $\delta\left(R, R^{\prime}\right) \geq 0$,

ii) Identity of indiscernibles: $\delta\left(R, R^{\prime}\right)=0$ if and only if $R=R^{\prime}$,

iii) Symmetry: $\delta\left(R, R^{\prime}\right)=\delta\left(R^{\prime}, R\right)$,

iv) Triangular Inequality: $\delta\left(R, R^{\prime \prime}\right) \leq \delta\left(R, R^{\prime}\right)+\delta\left(R^{\prime}, R^{\prime \prime}\right)$.

The following conditions numbered from 2 to 5 are introduced by Kemeny (1959). All of these conditions are also defined on each nonempty subset of $\mathcal{A}$, like Condition 1 . The following condition is the betweenness condition introduced in Kemeny (1959) as a strengthening of the triangular inequality condition.

Condition 2 (Betweenness): For all $R, R^{\prime}, R^{\prime \prime} \in \mathcal{W}$ such that $R^{\prime}$ is between $R$ and $R^{\prime \prime}$, we have:

$$
\delta\left(R, R^{\prime \prime}\right)=\delta\left(R, R^{\prime}\right)+\delta\left(R^{\prime}, R^{\prime \prime}\right)
$$

Remark 1. As also hinted in Kemeny and Snell (1962), note that an immediate consequence of betweenness for preferences on a line, e.g., $\left[R^{1}, \ldots, R^{t}\right]$ is the following: $\delta\left(R^{1}, R^{t}\right)=\delta\left(R^{1}, R^{2}\right)+$ $\delta\left(R^{2}, R^{3}\right)+\ldots+\delta\left(R^{t-2}, R^{t-1}\right)+\delta\left(R^{t-1}, R^{t}\right)$. In the main results, we shall often use such preferences on lines that are composed of consecutive elementary changes.

The next condition is the neutrality condition, which requires that the distances are neutral towards the names of the alternatives. Hence, a relabeling of these alternatives should not change

\footnotetext{
${ }^{1}$ Defining a metric only on a single subset would be analogous to, for instance, defining Euclidian distance only on $\mathbb{R}_{+}^{2}$ but not on, e.g., $\mathbb{R}_{+}^{5}$.
} 
the distance. Let $\pi: A \rightarrow A$ be a relabeling of the alternatives, i.e., a bijection, and denote $\pi R$ as the relabeled preference, i.e., for all $x, y \in A$ we have $\pi(x) R \pi(y)$ if and only if $x R y$. Formally:

Condition 3 (Neutrality): For all $R, R^{\prime} \in \mathcal{W}$ and for all relabeling of alternatives $\pi: A \rightarrow A$, we have:

$$
\delta\left(R, R^{\prime}\right)=\delta\left(\pi R, \pi R^{\prime}\right) .
$$

The following condition is known as normalization. This is a condition which simply restricts the minimal positive distance to be one. Kemeny (1959) suggests this condition as a choice of "a unit of measurement". Formally:

Condition 4 (Normalization):

$$
\min _{R, R^{\prime} \in \mathcal{W}}\left\{\delta\left(R, R^{\prime}\right): R \neq R^{\prime}\right\}=1 .
$$

The last condition, which is called reducibility, is a "variable alternative condition". It states that the distance should not be affected by deleting alternatives that are identically aligned at the top and/or bottom of the two preferences. Formally:

Condition 5 (Reducibility): For all $R, R^{\prime} \in \mathcal{W}$ such that for some $x, y \in A, R_{U C(x)}=R_{U C(x)}^{\prime}$ and $R_{L C(y)}=R_{L C(y)}^{\prime}$, we have:

$$
\delta\left(R, R^{\prime}\right)=\delta\left(R_{[x, y]}, R_{[x, y]}^{\prime}\right)
$$

\section{A new characterization}

We first reintroduce the definition of the swap distance (Kemeny, 1959$)$ and the well-known characterization of it below. Note that the definition and the characterization below is on the domain of weak preferences, i.e., preferences that allow ties. We omit here the superscript on weak preferences, e.g., $\mathcal{W}^{A}$, since the function is defined for any subset of alternatives $A \subseteq \mathcal{A}$.

Kemeny distance $\left(\delta_{K}\right):$ A function $\delta_{K}: \mathcal{W} \times \mathcal{W} \rightarrow \mathbb{R}$ is called the Kemeny distance whenever for all $R, R^{\prime} \in \mathcal{W}$, we have:

$$
\delta_{K}\left(R, R^{\prime}\right)=\left|R \backslash R^{\prime}\right|+\left|R^{\prime} \backslash R\right|
$$

Kemeny (1959) presents a characterization of the aforementioned distance function with the conditions in Section 3. The proof of this theorem, however, is provided in Kemeny and Snell (1962). We restate this theorem first:

Theorem. (Kemeny, 1959) A function $\delta: \mathcal{W} \times \mathcal{W} \rightarrow \mathbb{R}$ satisfies conditions 1, 2, 3, 4, and 5 if and only if $\delta$ is the Kemeny distance.

In this paper, we show that the conditions above are not logically independent, in particular "reducibility" (Condition 5) can be achieved as a byproduct of the other conditions imposed. This is not very straightforward to see in the proof of Kemeny and Snell (1962), since this condition is used in almost all lemmas involved in the construction of the proof for the main theorem. We do follow a somewhat different proof strategy without using this condition and provide a new characterization after Kemeny and Snell (1962) with minimal conditions. This implies that the first four conditions imply the fifth one, i.e., the reducibility condition. 
We first show that the first three conditions characterize a class of metrics that are Kemenylike. This class of distance functions comprises of multiples of the Kemeny distance with a constant $c^{*}$, since we do not impose normalization, just yet. In Theorem 1 we show that the first three conditions, i.e., metric, betweenness and neutrality characterize this Kemeny-like class on strict preferences. Thereafter we extend this result to all preferences, i.e., the weak orders (Theorem 2). Let $\delta$ be function satisfying Condition 1,2 , and 3 . Then, on the subdomain of strict preferences, $\delta$ is a Kemeny-like distance, i.e., for some positive constant $c \in \mathbb{R}_{++}, \delta=c^{*} \times \delta_{K}$. Formally;

Theorem 1. A function $\delta: \mathcal{L} \times \mathcal{L} \rightarrow \mathbb{R}$ satisfies conditions $1,2,3$ if and only if for some $c^{*} \in \mathbb{R}_{++}$ and for all $R, R^{\prime} \in \mathcal{L}$ :

$$
\delta\left(R, R^{\prime}\right)=c^{*} \times \delta_{K}\left(R, R^{\prime}\right)
$$

Proof. Let $E C^{k}$ denote the pairs of strict preferences which are elementary changes in position $k$. By neutrality, all elementary changes in the same position are assigned the same distance. Let us denote this distance correspondingly by $c_{k}$. We first show that for any two positions $k, l<m-1$, the distances are actually the same, i.e., $c_{k}=c_{l}=c$ for some positive real number $c$. Hence elementary changes are always assigned the same distance.

Take any two preferences $R, R^{\prime}$ such that both rank the alternatives identically except the $j^{t h}$, $(j+1)^{t h}$, and $(j+2)^{t h}$ alternatives as follows: $R=\ldots x y z \ldots$ and $R^{\prime}=\ldots z y x$. As the number of alternatives $m \geq 3$, one can always find such two preferences. Then there are two sequences of elementary changes that are "on a line" between $R$ and $R^{\prime}$ as in Remark 1:

- $\rho_{1}=[\ldots x y z \ldots, \ldots y x z \ldots, \ldots y z x \ldots, \ldots z y x \ldots]$,

- $\rho_{2}=[\ldots x y z \ldots, \ldots x z y \ldots, \ldots z x y \ldots, \ldots z y x \ldots]$.

By betweenness on two sequences, we have $\delta\left(R, R^{\prime}\right)=c_{j}+c_{j+1}+c_{j}=c_{j+1}+c_{j}+c_{j+1}$. Hence $c_{j}=c_{j+1}$. As the choice of position $j$ is arbitrary, this implies $c_{1}=c_{2}=\ldots=c_{m-1}=c$ for some real positive number $c$. As the choice of elementary change is also arbitrary, this implies for any elementary change at any position, we have $\delta\left(R, R^{\prime}\right)=c$. Since for elementary changes $\delta_{K}\left(R, R^{\prime}\right)=2$, this implies for any elementary change:

$$
\delta\left(R, R^{\prime}\right)=\frac{c}{2} \times \delta_{K}\left(R, R^{\prime}\right)
$$

Finally, consider any distinct $R, R^{\prime}$ and let $\left|R \backslash R^{\prime}\right|=t$ for some positive integer $t$. Consider any sequence of elementary changes $\rho=[\rho(1), \rho(2), \ldots, \rho(t), \rho(t+1)]$ which is on a line between $R$ and $R^{\prime}$. By Condition 2, we have $\delta\left(R, R^{\prime}\right)=\delta(\rho(1), \rho(2))+\ldots+\delta(\rho(t), \rho(t+1))=c+\ldots+c(t$ times). Hence $\delta\left(R, R^{\prime}\right)=c \times t=c \times\left|R \backslash R^{\prime}\right|$. Since $\left|R \backslash R^{\prime}\right|=\left|R^{\prime} \backslash R\right|$, Equation 1 implies for any two distinct preferences:

$$
\delta\left(R, R^{\prime}\right)=\frac{c}{2} \times \delta_{K}\left(R, R^{\prime}\right)
$$

Setting $c^{*}=c / 2$ completes the proof since for other cases, i.e., $R=R^{\prime}$ we have $\delta\left(R, R^{\prime}\right)=$ $\delta_{K}\left(R, R^{\prime}\right)=0$ by the metric condition.

Next we show that the characterization in Theorem 1 still works on the larger domain of weak preferences.

Theorem 2. A function $\delta: \mathcal{W} \times \mathcal{W} \rightarrow \mathbb{R}$ satisfies conditions $1,2,3$ if and only if for some $c^{*} \in \mathbb{R}_{++}$ and for all $R, R^{\prime} \in \mathcal{W}$ :

$$
\delta\left(R, R^{\prime}\right)=c^{*} \times \delta_{K}\left(R, R^{\prime}\right)
$$


Proof. The "if part" can be found in Kemeny and Snell (1962). The see the "only if" part, let $\delta$ satisfy the conditions. By Theorem 1, the statement is true if both preferences are strict.

Case 1: Consider any $P \in \mathcal{L}$ and any $R \in \mathcal{W} \backslash \mathcal{L}$, i.e, one preference is strict. We construct two new strict preferences as follows:

$$
\begin{aligned}
& P_{R}^{+}=\{(x, y) \in R:(y, x) \notin R\} \cup\{(x, y) \in R:(y, x) \in R \backslash P\} \cup\{(x, y) \in R: x=y\} \\
& P_{R}^{-}=\{(x, y) \in R:(y, x) \notin R\} \cup\{(x, y) \in R:(y, x) \in R \cap P\}
\end{aligned}
$$

Verbally, $P_{R}^{+}$is the strict extension of the weak preference $R$ in line with $P$, whereas $P_{R}^{-}$ is, roughly speaking, the mirror image of $P_{R}^{+}$with $R$ in the center. Hence, by construction, we have $\left[P, P_{R}^{+}, R, P_{R}^{-}\right]$on a line between $P$ and $P_{R}^{-}$. We refer the reader to Example 1 for such a construction. By betweenness, we have the following two equations:

$$
\begin{gathered}
\delta\left(P, P_{R}^{-}\right)=\delta\left(P, P_{R}^{+}\right)+\delta\left(P_{R}^{+}, R\right)+\delta\left(R, P_{R}^{-}\right) \\
\delta(P, R)=\delta\left(P, P_{R}^{+}\right)+\delta\left(P_{R}^{+}, R\right)
\end{gathered}
$$

Because of neutrality, $\delta\left(P_{R}^{+}, R\right)=\delta\left(P_{R}^{-}, R\right)$. Since $\delta$ is a metric, by symmetry $\delta\left(P_{R}^{+}, R\right)=\delta\left(R, P_{R}^{-}\right)$. By Equation 3, we have:

$$
2 \times \delta\left(P_{R}^{+}, R\right)=\delta\left(P, P_{R}^{-}\right)-\delta\left(P, P_{R}^{+}\right)
$$

Plugging this in Equation 4, we have:

$$
\delta(P, R)=\delta\left(P, P_{R}^{+}\right)+\frac{1}{2} \times\left(\delta\left(P, P_{R}^{-}\right)-\delta\left(P, P_{R}^{+}\right)\right)
$$

By Theorem 1, there exists $c^{*} \in \mathbb{R}_{++}$such that:

$$
\begin{gathered}
\delta(P, R)=c^{*} \times \delta_{K}\left(P, P_{R}^{+}\right)+\frac{1}{2} \times\left(c^{*} \times \delta_{K}\left(P, P_{R}^{-}\right)-c^{*} \times \delta_{K}\left(P, P_{R}^{+}\right)\right) \\
=\frac{1}{2} \times c^{*} \times\left(\delta_{K}\left(P, P_{R}^{-}\right)+\delta_{K}\left(P, P_{R}^{+}\right)\right)
\end{gathered}
$$

Finally by betweenness, this implies

$$
\delta(P, R)=c^{*} \times \delta_{K}(P, R)
$$

Case 2: Consider any $R, \bar{R} \in \mathcal{W} \backslash \mathcal{L}$, i.e., both preferences are weak. Let $P \in \mathcal{L}$ be any strict preference. We construct two new strict preferences based on $P$ as follows:

$$
\begin{aligned}
& P_{R}^{+}=\{(x, y) \in R:(y, x) \notin \bar{R}\} \cup\{(x, y) \in R:(y, x) \in R \backslash \bar{R}\} \cup\{(x, y) \in R:(y, x) \in \\
& (R \cap \bar{R}) \backslash P\} \cup\{(x, y) \in R: x=y\} \\
& P_{\bar{R}}^{-}=\{(x, y) \in \bar{R}:(y, x) \notin \bar{R}\} \cup\{(x, y) \in \bar{R}:(y, x) \in \bar{R} \backslash R\} \cup\{(x, y) \in \bar{R}:(y, x) \in \\
& (R \cap \bar{R} \cap P)\}
\end{aligned}
$$

Verbally, $P_{R}^{+}$is the strict extension of $R$ towards $P$ and away from $\bar{R}$, whereas $P_{\bar{R}}^{-}$is the strict extension $\bar{R}$ towards the inverse $\bigsqcup^{2}$ of $P$ and away from $R$. Hence, by construction, we have $\left[P_{R}^{+}, R, \bar{R}, P_{\bar{R}}^{-}\right]$on a line between $P_{R}^{+}$and $P_{\bar{R}}^{-}$. By betweenness, we have the following equation:

\footnotetext{
${ }^{2}$ By inverse of a preference we mean a inversion of each pair in a binary relation, e.g., if $P=a b c$ then the inverse of $P$ is denoted by $P_{I}=c b a$.
} 


$$
\delta\left(P_{R}^{+}, P_{\bar{R}}^{-}\right)=\delta\left(P_{R}^{+}, R\right)+\delta(R, \bar{R})+\delta\left(\bar{R}, P_{\bar{R}}^{-}\right)
$$

By Theorem 1 and Case 1 , there exists $c^{*} \in \mathbb{R}_{++}$such that:

$$
\delta\left(P_{R}^{+}, P_{\bar{R}}^{-}\right)=c^{*} \times \delta_{K}\left(P_{R}^{+}, P_{\bar{R}}^{-}\right)=c^{*} \times \delta_{K}\left(P_{R}^{+}, R\right)+\delta(R, \bar{R})+c^{*} \times \delta_{K}\left(\bar{R}, P_{\bar{R}}^{-}\right)
$$

Then,

$$
\delta(R, \bar{R})=c^{*} \times \delta_{K}\left(P_{R}^{+}, P_{\bar{R}}^{-}\right)-c^{*} \times \delta_{K}\left(P_{R}^{+}, R\right)-c^{*} \times \delta_{K}\left(\bar{R}, P_{\bar{R}}^{-}\right)
$$

Finally by betweenness, this implies

$$
\delta(R, \bar{R})=c^{*} \times \delta_{K}(R, \bar{R})
$$

Now that we have shown that Conditions 1, 2, and 3 characterize the class of multiples of the Kemeny distance, we can introduce the normalization and provide the re-characterization as a corollary.

Corollary 1. A function $\delta: \mathcal{W} \times \mathcal{W} \rightarrow \mathbb{R}$ satisfies conditions $1,2,3$ and 4 if and only if for all $R, R^{\prime} \in \mathcal{W}:$

$$
\delta\left(R, R^{\prime}\right)=\delta_{K}\left(R, R^{\prime}\right)
$$

Proof. By normalization of the Kemeny distance, the minimal Kemeny distance is 1 . This implies for some $R, R^{\prime} \in \mathcal{W}, \delta_{K}\left(R, R^{\prime}\right)=1$. By Theorem 2 , we have $\delta=c^{*} \times \delta_{K}$. Then $\delta\left(R, R^{\prime}\right)=c^{*} \times 1$. Since $\delta$ is an increasing function of $\delta_{K}, \delta\left(R, R^{\prime}\right)$ should be minimal too. By normalization of $\delta$, this implies $\delta\left(R, R^{\prime}\right)=c^{*}=1$. Hence $\delta=\delta_{K}$

\section{Conclusion}

\subsection{Discussion}

In his seminal book, Kemeny and Snell (1962) proposes three possibilities regarding a characterization of any function with reasonable conditions, e.g., conditions mentioned in Section 3 ;

In general, given a set of axioms for an unknown quantity, such as $\delta$, there are three possibilities: (I) The axioms are inconsistent; i.e., there is no $\delta$ satisfying all axioms.

(II) There is a uniqe $\delta$ satisfying all axioms. (III) There is more than one $\delta$.

In this paper, we point to the fourth possibility where the conditions may be unnecessarily too many, i.e., they may not be logically independent. This may make a function look too demanding in terms of conditions it satisfies. However, as Theorems 1 and 2 together with Corollary 1 show, the reducibility condition is not necessary for the characterization of the Kemeny distance. This means the Kemeny distance is in fact less demanding than it was assumed to be since Kemeny $(1959)$. Next, we conclude the paper to show that conditions of the new characterization we propose are indeed logically independent. 


\subsection{Logical independence of conditions}

To discuss the logical independence of the conditions used in the characterization, consider the following four functions:

(i) Biased Kemeny distance: For some fixed alternative $a \in A$ predefined for each $A \in P(\mathcal{A})$, and for all $R, R^{\prime} \in \mathcal{W}^{A}$ :

$$
\delta_{B K}\left(R, R^{\prime}\right)=\#\left[\left(R \backslash R^{\prime}\right) \cup\left(R^{\prime} \backslash R\right)\right]+\#\left[\left(R \backslash R^{\prime}\right) \cup\left(R^{\prime} \backslash R\right) \cap(\{a\} \times A \cup A \times\{a\})\right]
$$

(ii) Discrete distance: For all $R, R^{\prime} \in \mathcal{W}$ :

$$
\delta_{D}\left(R, R^{\prime}\right)= \begin{cases}1, & \text { if } R \neq R^{\prime} \\ 0, & \text { if } R=R^{\prime}\end{cases}
$$

(iii) Scaled Kemeny distance: For all $R, R^{\prime} \in \mathcal{W}$ :

$\delta_{S K}\left(R, R^{\prime}\right)=2 \cdot \delta_{K}\left(R, R^{\prime}\right)$

(iv) Null-Kemeny function: For some fixed $A=\{a, b\} \in P(\mathcal{A})$, let us define $R_{1}=a b, R_{2}=b a$ and let $\bar{R}$ be such that $a$ and $b$ are ordered equally good. Then for all $R, R^{\prime} \in \mathcal{W}$ :

$$
\alpha_{N K}\left(R, R^{\prime}\right)= \begin{cases}1, & \text { if }\left(R, R^{\prime}\right) \in\left\{\left(R_{1}, \bar{R}\right),\left(R_{2}, \bar{R}\right)\right\} \\ 2, & \text { if }\left(R, R^{\prime}\right) \in\left\{\left(\bar{R}, R_{1}\right),\left(\bar{R}, R_{2}\right)\right\} \\ 3, & \text { if }\left(R, R^{\prime}\right)=\left(R_{1}, R_{2}\right) \\ \delta_{K}\left(R, R^{\prime}\right), & \text { otherwise }\end{cases}
$$

The biased Kemeny distance (i) weighs the elementary changes which involve alternative $a$ twice. The discrete distance (ii) is a well-known example which indicates whether orders are different or not. The scaled Kemeny distance (iii) scales the Kemeny distance by a factor of 2. Null-Kemeny function (iv) is almost identical to the Kemeny distance except for when preferences are over a fixed subset of 2 alternatives.

It is straightforward to prove that the first three (i, ii, and iii) are distance functions whereas the Null-Kemeny function is not (it fails symmetry of Condition 1). First, (i) $\delta_{B K}$ satisfies betweenness and normalization but is not neutral. Second, (ii) $\delta_{D}$ satisfies neutrality and normalization but not betweenness. Third, (iii) $\delta_{S K}$ satisfies betweenness and neutrality but not normalization. Finally, (iv) $\alpha_{N K}$ satisfies all conditions except metric conditions since it is not symmetric, and therefore is not a distance function at all. Therefore, these four functions together show that the characterizing conditions in Theorems 1 and 2 , and in Corollary 1 are logically independent.

We conclude our paper by providing an example of the construction in Case 1 in the proof of Theorem 2 ,

Example 1. Let $A=\{a, b, c\}$. Consider the following preferences $P \in \mathcal{L}, R \in \mathcal{W}$ and the path constructed for Case 1 in the proof of Theorem 2.

$\left.\begin{array}{cccccc}P & R & {[P,} & P_{R}^{+}, & R, & P_{R}^{-}\end{array}\right]$




\section{References}

Bogart, K. (1973). Preference structures I: Distances between transitive preference relations. Journal of Mathematical Sociology 3(1), 49-67.

Cramer, G. (1750). Introduction à l'analyse des lignes courbes algébriques. Genève: Frères Cramer et C. Philibert.

Damerau, F. (1964). A technique for computer detection and correction of spelling errors. Communications of the ACM 7(3), 171-176.

Hamming, R. (1950). Error detecting and error correcting codes. Bell System Technical Journal 29(2), 147-160.

Kemeny, J. (1959). Mathematics without numbers. Daedalus 88(4), 577-591.

Kemeny, J. and J. Snell (1962). Mathematical models in the social sciences. Boston: Ginn.

Kendall, M. (1938). A new measure of rank correlation. Biometrika 30(1/2), 81-93.

Levenshtein, V. (1966). Binary codes capable of correcting deletions, insertions, and reversals. In Soviet Physics Doklady, Volume 10, pp. 707-710. 